\title{
Association of Atrial Fibrillation with Morphological and Electrophysiological Changes of the Atrial Myocardium
}

\author{
Adéla Matějková , Ivo Šteiner \\ Charles University, Faculty of Medicine and University Hospital in Hradec Králové, Czech Republic: Fingerland Depart- \\ ment of Pathology \\ * Corresponding author: Fingerland Department of Pathology, Charles University, Faculty of Medicine and Faculty Hospital \\ in Hradec Králové, Sokolská 581, 50005 Hradec Králové, Czech Republic; email: adela.matejkova@gmail.com
}

\begin{abstract}
Summary: Atrial fibrillation (AF) is the most common sustained cardiac arrhythmia. For long time it was considered as pure functional disorder, but in recent years, there were identified atrial locations, which are involved in the initiation and maintenance of this arrhythmia. These structural changes, so called remodelation, start at electric level and later they affect contractility and morphology. In this study we attempted to find a possible relation between morphological (scarring, amyloidosis, left atrial (LA) enlargement) and electrophysiological (ECG features) changes in patients with AF. We examined grossly and histologically 100 hearts of necropsy patients - 54 with a history of AF and 46 without AF. Premortem ECGs were evaluated. The patients with AF had significantly heavier heart, larger LA, more severely scarred myocardium of the LA and atrial septum, and more severe amyloidosis in both atria. Severity of amyloidosis was higher in LAs vs. right atria (RAs). Distribution of both fibrosis and amyloidosis was irregular. The most affected area was in the LA anterior wall. Patients with a history of AF and with most severe amyloidosis have more often abnormally long P waves. Finding of long $\mathrm{P}$ wave may contribute to diagnosis of a hitherto undisclosed atrial fibrillation.
\end{abstract}

Keywords: Atrial fibrillation; Isolated atrial amyloid; Myocardial scarring; Electrocardiographic features; P wave

\section{Introduction}

Atrial fibrillation (AF) is the most common sustained cardiac arrhythmia in the group of supraventricular arrhythmias $(7,11)$. Although it does not directly endanger the patient's life, it certainly cannot be regarded as benign. In the Czech Republic, AF is the commonest arrhythmia leading to hospitalization (16). There are several complications associated with AF, particularly thromboembolism, more common heart failure and poorer quality of life. Patients with AF have markedly increased mortality. In developed countries, the prevalence of AF is $1-2 \%$ (11). With ageing, its prevalence significantly rises. In the fifth decennium it is $1 \%$ and doubles in each sequential decennium, reaching more than $10 \%$ in octogenerians $(7,16)$. For subjects aged $40+$ years, regardless of gender, the risk of AF is estimated at $25 \%$ (15).

AF is a complex arrhythmia. It affects a wide spectrum of individuals, both with and without structural heart diseases. The key risk factors for AF include, in addition to age, arterial hypertension, congestive heart failure and valvular diseases (7). In AF, the heart rhythm is not controlled by the sinoatrial node, but by multiple ectopic pacemakers in the atrial chambers, resulting in chaotic depolarization of atrial myocardium, which eventually becomes sustained by local reentrant circuits. The most common site for triggering premature atrial contraction are foci in atrial walls, particularly in so called myocardial sleeves of pulmonary veins $(7,9,10)$.

The key etiopathogenic factor of AF seems to be restructuring (remodelation) of the atrial myocardium leading to atrial conduction abnormalities. This remodelation starts at electric level and later it affects contractility and structure (1). The structural changes are both geometric-dilatation of the atria, and anatomo-histological - fibrosis and amyloidosis of atrial myocardium, particularly deposition of isolated atrial amyloid (IAA). The incidence of both AF and IAA is related to ageing $(2,7,21)$. The dominant heart chamber for development of AF is the left atrium (LA); the right atrium (RA) plays a minor role. There exists a vicious circle - the once developed AF is self-worsening, as it leads to more structural rebuilding of the atrial myocardium and this is associated with progression of AF into its more sustained forms (19).

In this study, in cooperation with clinicians-cardiologists, we attempted to find possible relation between morphological changes of atrial myocardium and electrophysiological (ECG) abnormalities of atrial conduction, by comparing two groups of necropsy patients - those with AF and those without AF (control group). Particular attention was paid to cases with heavy amyloid infiltration of the atrial myocardium. 


\section{Material and Methods}

During 2007-2011 we examined 100 hearts of patients autopsied at the Fingerland Department of Pathology in Hradec Králové. The criteria required for inclusion in the study were patient's age 50-90 years, and an available ECG record from less than 3 months before death. The study was approved by the Ethical Committee of Faculty Hospital in Hradec Králové.

The total of 100 patients studied comprised two main groups - AF group - 54 patients with a history of AF, and SINUS group -46 patients with a proved sinus rhythm. The groups were age- and sex-matched. The AF group was further subclassified into two subgroups: subgroup AFF patients with AF present on their last ECG, and subgroup AFS - patients with a history of AF who, however, had sinus rhythm on their last ECG. Information regarding the type of AF was retrospectively obtained from the patient's clinical documentation. The subgroup AFF $(n=25)$ comprised patients with the following types of AF: 2 newly diagnosed, 2 paroxysmal, 4 persistent, 5 permanent, 7 described as "chronic", and 5 not specified. Analogically, the subgroup AFS $(n=29)$ comprised patients with 6 newly diagnosed, 15 paroxysmal, 3 permanent, 3 described as "chronic", and 2 not specified type of AF.

At gross examination of the formalin-fixed hearts attention was paid particularly to the LA. Its interior was measured in 3 dimensions and its volume calculated. Further, we related the LA volume to the patient's body surface area (BSA) by the Du Bois formula: BSA $=$ (body weight ${ }^{0.425}$ $\times$ height $\left.t^{0.725}\right) \times 0.007184$. This calculation is used in ultrasound examination of the heart to assess the LA size (17).

For histological examination, we obtained five samples from standard sites: RA, 3 samples from the LA - anterior wall (LAA), roof (LAR), and posterior wall (LAP), and interatrial septum (IAS). All samples were processed in a standard way and sections stained by haematoxylin and eosin, by Sirius red (Maldyk) for amyloid, and by Elastica - Van Gieson for fibrous scarring. The degree of IAA deposition in the atrial walls was semiquantitatively graded for each heart on a $0-3$ scale according to the following criteria: grade $0=$ not present or only small deposits; grade $1=$ occasional fine fibers surrounding cardiomyocytes and/or deposited in the walls of small intramyocardial vessels; grade $2=$ moderate deposits in the entire thickness of the myocardium; grade 3 = dense network of fibers and/or solid foci of amyloid.

Similarly, the degree of fibrosis (scarring) was assessed: grade $0=$ absence or focal loose interstitial fibrosis; grade $1=$ mild diffuse interstitial fibrosis or few small solid foci; grade $2=$ moderate degree of interstitial fibrosis or more solid foci in the entire thickness of the myocardium; grade $3=$ severe interstitial fibrosis or large solid foci.

The patient's baseline 12 - lead ECGs, obtained in the supine position usually just prior to death with $25 \mathrm{~mm} / \mathrm{s}$ and $10 \mathrm{~mm} / \mathrm{mV}$ standardization, were screened by Vignendra Ariyarajah, MD (Saint Boniface Hospital, Winnipeg, Man.,
Canada) and Jiří Nový, MD (Hospital in Jičín, Faculty Hospital in Hradec Králové). ECGs were assessed with a calibrated, magnifying graticule, in the same way as in the previous study of Ariyarajah et al. (2). The following parameters were assessed: $\mathrm{P}$ wave duration (P max, $\mathrm{P}$ min), $\mathrm{P}$ wave dispersion ( $\mathrm{P}$ disp - difference in duration between the widest (maximum) and the narrowest (minimum) $\mathrm{P}$ wave), $\mathrm{P}$ wave axis (P axis), duration of QRS complex (QRS max, QRS min), QRS complex dispersion (QRS disp - difference between the widest and the narrowest QRS complex), and the heart electric axis (QRS axis).

All the results (gross LA parameters, grades of fibrosis and of amyloid deposition) were compared in the AF vs. SINUS groups, and also in both AF subgroups - AFF and AFS vs. SINUS, and AFF vs. AFS.

For evaluation of influence of the myocardial structural changes, particularly the infiltration by IAA on atrial conduction, we compared ECG findings of the group IAA grade 0

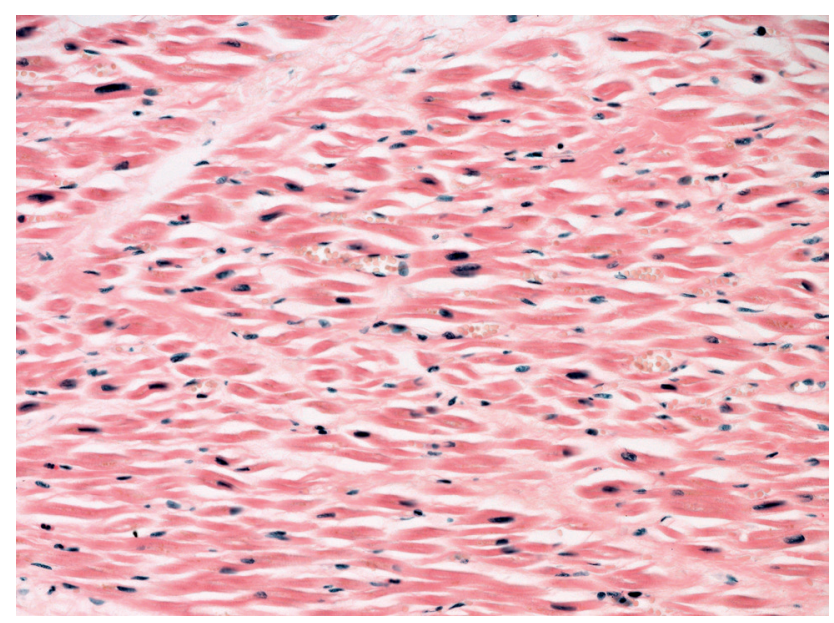

Fig. 1: Sirious red staining of left atrial anterior wall showing IAA grade 0 (amyloid is not present). Magnificantion 200×.

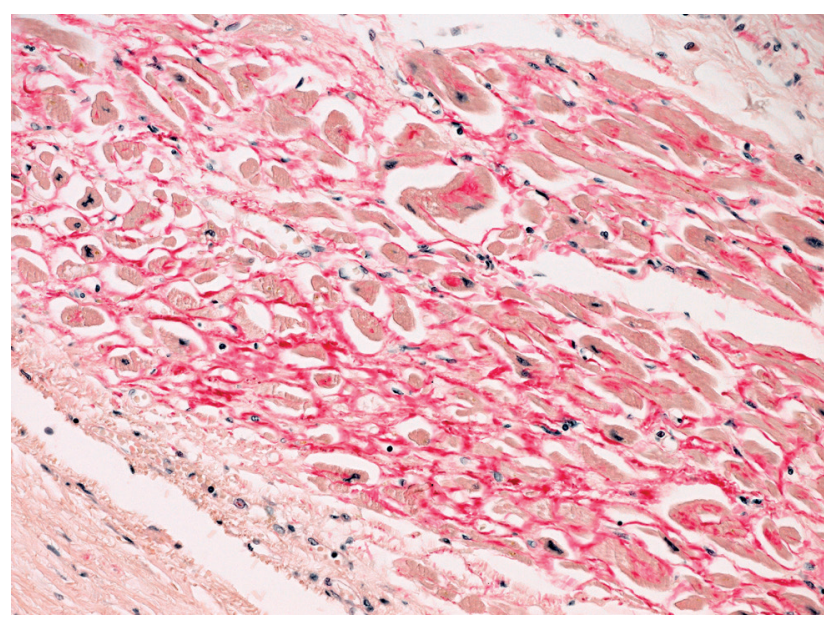

Fig. 2: Sirious red staining of left atrial anterior wall showing IAA grade 3 (dense network of amyloid fibers). Magnificantion 200×. 
(fig. 1) with those of IAA grade 3 (fig. 2). (To increase specificity, the IAA groups 1 and 2 were omitted.) For this comparison we used samples from LAA. (In our previous study, this showed significantly most intense infiltration by amyloid (18).) In control cases with sinus rhythm we evaluated features of both $\mathrm{P}$ wave and QRS complex. In cases with AF, only QRS complex was evaluated.

The data obtained were evaluated statistically by multiple appropriate tests: two - sample t test, Mann Whitney's U test, Fisher's LSD Multiple-Comparison Test, Kruskal-Wallis Multiple-Comparison Z-Value Test (Dunn`s Test), Fisher's exact test or chi-square test of independence in contingency tables. Quantitative parameters were presented by median with $95 \%$ confidence interval. A value of $\mathrm{p} \leq 0.05$ was considered to be statistically significant.

\section{Results}

Our series of 100 subjects comprised 51 men and 49 women. In the main AF group (54 subjects), there were 29 men and 25 women, with median of age 75 (70-77) years; in the control group SINUS (46 subjects), there were 22 men and 24 women, with median of age $66(62-74)$ years. The AF subgroups AFF and AFS comprised 25 subjects (13 men and 12 women) with median of age 74 (69-76) years, and 29 subjects (16 men and 13 women) with median of age 76 (68-80) years, respectively. Baseline characteristics of the groups obtainable at the time of autopsy (age, gender, presence of systemic hypertension, diabetes mellitus, coronary artery disease, mitral stenosis or artificial heart valves) are presented in table 1. Hypertension, diabetes mellitus and mitral valve disease occurred more frequently in patients with AF. Statistically significant difference between groups was obvious only in age $(p=0.03)$ and systemic hyperten$\operatorname{sion}(\mathrm{p}=0.01)$.

There were significant differences $(p<0.001)$ in heart weight between the AF and control group; in the AFs the median heart weight was $525 \mathrm{~g} \mathrm{(480-590} \mathrm{g),} \mathrm{while} \mathrm{in} \mathrm{con-}$ trols $420 \mathrm{~g}$ (390-490 g). Also comparison of the two AF subgroups (AFF, AFS) with the control group showed statistically significant differences $(p<0.001)$. This was most marked with the AFF where the median heart weight was $560 \mathrm{~g}(470-680 \mathrm{~g})$.

Statistically significant difference between the two main groups $(\mathrm{p}=0.002)$ was noted in the LA volume related to body surface; subjects with AF had more voluminous LA: median 87 (79-101) ml than those with sinus rhythm: median 66 (60-76) $\mathrm{ml}$. The same applied to the subgroups AFF and AFS vs. SINUS ( $p=0.004)$. The most significant difference was between AFF: median 92 (79-114) $\mathrm{ml}$ and controls.

Histological examination showed significantly more severe myocardial fibrosis in all samples of LA walls and interatrial septum in the AF group when compared to controls. In the RA, however, the difference was not statistically significant. Most severe scarring was observed in the AFF subgroup (tab. 2a).

Similar differences were noted in the degree of myocardial deposition of amyloid. More severe amyloidosis was observed in the AF than in the SINUS group, with the AFF subgroup most affected (tab. 2b).

In all groups the severity of amyloid deposits was higher in the LA compared to the RA. In majority of the evaluated areas the difference was statistically significant, except for two comparisons, however, even there the difference was obvious (tab. 3).

Tab. 1: Baseline characteristics of the groups.

\begin{tabular}{|c|c|c|c|c|c|}
\hline & SINUS $(n=46)$ & $\mathrm{AF}(\mathrm{n}=54)$ & $\operatorname{AFS}(n=29)$ & $\operatorname{AFF}(n=25)$ & p value \\
\hline $\begin{array}{l}\text { Age (median with } 95 \% \\
\text { confidence interval), years }\end{array}$ & $66(62-74)$ & $75(70-77)$ & $76(68-80)$ & $74(69-76)$ & $0.03 *$ \\
\hline Gender - Male/Female & $22 / 24$ & $29 / 25$ & $16 / 13$ & $13 / 12$ & 0.82 \\
\hline Systemic hypertension & $32(70)$ & $50(93)$ & $26(90)$ & $24(96)$ & $0.01 *$ \\
\hline Diabetes mellitus & $16(35)$ & $24(44)$ & $12(41)$ & $12(48)$ & 0.55 \\
\hline Coronary artery disease $^{1}$ & & & & & 0.42 \\
\hline$\leq 25 \%$ & $19(41)$ & $11(20)$ & $6(21)$ & $5(20)$ & \\
\hline $26-50 \%$ & $8(17)$ & $15(28)$ & $9(31)$ & $6(24)$ & \\
\hline $51-75 \%$ & $4(9)$ & $9(17)$ & $5(17)$ & $4(16)$ & \\
\hline$>75 \%$ & $15(33)$ & $19(35)$ & $9(31)$ & $10(40)$ & \\
\hline Mitral stenosis & $6(13)$ & $10(19)$ & $7(24)$ & $3(12)$ & - \\
\hline Artificial mitral valve & $0(0)$ & $3(6)$ & $3(10)$ & $0(0)$ & - \\
\hline
\end{tabular}

All values represent numbers of patients with percentages in parentheses unless indicated otherwise.

$\mathrm{n}=$ number of hearts in individual group or subgroup. ${ }^{1}$ Degree of atherosclerotic luminal stenosis at autopsy.

$* \mathrm{p} \leq 0.05$ considered as significant difference. 
Tab. 2: Two - sample and three - sample comparison of atrial myocardial structural changes.

a) Severity of scarring in particular areas of the atria.

\begin{tabular}{|l|c|c|c|c|c|c|c|}
\hline SCARRING & AF $(\mathbf{n}=\mathbf{5 4})$ & SINUS $(\mathbf{n}=\mathbf{4 6})$ & $\boldsymbol{p}$ & AFS $(\mathbf{n}=\mathbf{2 9})$ & AFF $(\mathbf{n}=\mathbf{2 5})$ & SINUS (n= 46) & $\boldsymbol{p}$ \\
\hline RA & 1.46 & 1.46 & $n . s$. & 1.62 & 1.28 & 1.46 & $n . s$. \\
\hline LAA & 1.72 & 1.11 & 0.004 & 1.55 & 1.92 & 1.11 & 0.010 \\
\hline LAR & 1.70 & 1.04 & $<0.001$ & 1.48 & 1.96 & 1.04 & $<0.001$ \\
\hline LAP & 1.59 & 1.15 & 0.001 & 1.45 & 1.76 & 1.15 & 0.001 \\
\hline IAS right & 1.26 & 0.74 & 0.001 & 1.21 & 1.32 & 0.74 & 0.003 \\
\hline IAS left & 1.63 & 1.07 & 0.010 & 1.45 & 1.84 & 1.07 & 0.006 \\
\hline
\end{tabular}

b) Severity of amyloid infiltration in particular areas of the atria.

\begin{tabular}{|l|c|c|c|c|c|c|c|}
\hline AMYLOID & AF $(\mathbf{n}=\mathbf{5 4})$ & SINUS $(\mathbf{n}=\mathbf{4 6})$ & $\boldsymbol{p}$ & AFS $(\mathbf{n}=\mathbf{2 9})$ & AFF $(\mathbf{n}=\mathbf{2 5})$ & SINUS $(\mathbf{n}=\mathbf{4 6})$ & $\boldsymbol{p}$ \\
\hline RA & 1.56 & 0.98 & 0.002 & 1.34 & 1.80 & 0.98 & 0.001 \\
\hline LAA & 2.39 & 1.59 & 0.005 & 2.31 & 2.48 & 1.59 & 0.030 \\
\hline LAR & 1.77 & 1.22 & 0.007 & 1.72 & 1.83 & 1.22 & 0.052 \\
\hline LAP & 2.02 & 1.22 & 0.003 & 2.00 & 2.04 & 1.22 & 0.023 \\
\hline IAS right & 0.70 & 0.50 & 0.003 & 0.69 & 0.72 & 0.50 & 0.003 \\
\hline IAS left & 1.96 & 1.48 & 0.010 & 1.97 & 1.96 & 1.48 & $n . s$. \\
\hline
\end{tabular}

The tables show average grades of scarring and amyloid infiltration in the heart atria. For statistical evaluation, the Fisher's exact test and chi-square test of independence in contingency tables were used. A value of $p \leq 0.05$ was considered to be statistically significant. $\mathrm{n}=$ number of hearts; $\mathrm{n} . \mathrm{s} .=$ no significant difference.

Tab. 3: Comparison of right vs. left atrial amyloidosis in individual groups.

\begin{tabular}{|l|c|c|c|c|c|c|}
\hline AMYLOID & RA & LAA & $\boldsymbol{p}$ & IAS right & IAS left & $\boldsymbol{p}$ \\
\hline SINUS (n= 46) & 0.98 & 1.59 & 0.001 & 0.50 & 1.48 & $<0.001$ \\
\hline AF $(\mathbf{n}=\mathbf{5 4})$ & 1.56 & 2.39 & 0.003 & 0.70 & 1.96 & $<0.001$ \\
\hline AFS (n= 29) & 1.34 & 2.31 & $n . s$. & 0.69 & 1.97 & $<0.001$ \\
\hline AFF $(\mathbf{n}=\mathbf{2 5})$ & 1.80 & 2.48 & 0.050 & 0.72 & 1.96 & n.s. \\
\hline
\end{tabular}

The table shows average grades of amyloidosis in particular areas of heart atria. For statistical evaluation, the Fisher's exact test and chi-square test of independence in contingency tables were used. A value of $\mathrm{p} \leq 0.05$ was considered to be statistically significant. $\mathrm{n}=$ number of hearts; n.s. = no significant difference.

The severity of both myocardial fibrosis and amyloidosis was compared in the three systematically examined sites of the left atrium (LAA, LAP and LAR). While severity of fibrosis was not significantly different, amyloidosis showed statistically significant differences $(\mathrm{p}<0.025-0.001)$ in all groups (AF, SINUS, AFF and AFS) - it was most intense in the LA anterior wall, followed by LA posterior wall and the roof of the LA.

To check possible impact of structural changes of atrial myocardium on its conduction properties we compared ECG characteristics of the group with no amyloid (grade 0 ) with the one with most severe amyloidosis (grade 3). Thus, we evaluated 62 ECG records (16 with IAA grade 0 and 46 with IAA grade 3). Of the total 62 cases, 39 featured sinus rhythm, and $23 \mathrm{AF}$. There was no statistically significant dif- ference in any of the parameters followed between $\mathrm{AF}$ and the control group (tab. 4). Further, we examined frequency of the abnormally long P waves (Pmax $\geq 120 \mathrm{~ms}$ ) between the groups IAA 0 and IAA 3 . Long P waves were seen mostly in the IAA 3 group. When comparing this parameter in the subgroup AFS and the controls, long P waves were clearly more common in subjects with history of AF ( $46 \%$ vs. $14 \%$ ) (tab. 5).

\section{Discussion}

Currently, it is generally accepted that AF has a morphological basis and is associated with remodelation of the atrial myocardium $(1,21,23)$. This remodelation is based on myocardial injury of various pathogenesis. The two main 
Tab. 4: Comparison of ECG features in IAA 0 vs. IAA 3.

\begin{tabular}{|l|c|c|c|}
\hline P wave & $\begin{array}{c}\text { IAA 0 } \\
(\mathbf{n}=\mathbf{1 2})\end{array}$ & $\begin{array}{c}\text { IAA 3 } \\
(\mathbf{n}=\mathbf{2 7})\end{array}$ & $\boldsymbol{p}$ \\
\hline P max (ms) & $100(90 ; 120)$ & $100(80 ; 120)$ & 0.459 \\
\hline P min (ms) & $55(40 ; 80)$ & $60(50 ; 70)$ & 0.836 \\
\hline P disp (ms) & $40(30 ; 50)$ & $40(30 ; 50)$ & 0.356 \\
\hline P axis ( $\left(^{\circ}\right.$ & $50(30 ; 70)$ & $50(45 ; 70)$ & 0.417 \\
\hline \hline QRS complex & $\begin{array}{c}\text { IAA 0 } \\
(\mathbf{n}=\mathbf{1 6})\end{array}$ & $\begin{array}{c}\text { IAA 3 } \\
(\mathbf{n}=\mathbf{4 6})\end{array}$ & $\boldsymbol{p}$ \\
\hline QRS max (ms) & $105(90 ; 120)$ & $100(90 ; 100)$ & 0.682 \\
\hline QRS min (ms) & $65(50 ; 80)$ & $70(60 ; 80)$ & 0.490 \\
\hline QRS disp (ms) & $40(30 ; 50)$ & $30(20 ; 30)$ & 0.067 \\
\hline QRS axis $\left({ }^{\circ}\right)$ & $25(-40 ; 60)$ & $0(-5 ; 20)$ & 0.442 \\
\hline
\end{tabular}

The tables show median values of ECG characteristics with a $95 \%$ confidence interval. A value of $p \leq 0.05$ was considered to be statistically significant. $\mathrm{n}=$ number of hearts.

Tab. 5: Comparison of "long P wave" frequency in IAA 0 vs. IAA 3 , and in AFS vs. SINUS.

\begin{tabular}{|l|c|c|}
\hline P $\max \geq \mathbf{1 2 0}(\mathbf{m s})$ & IAA 0 $(\mathbf{n}=\mathbf{1 2})$ & IAA 3 $(\mathbf{n}=\mathbf{2 6})$ \\
\hline Total $(\mathbf{1 2} / \mathbf{3 8})$ & $3(25)$ & $9(35)$ \\
\hline AFS $(\mathbf{9} / \mathbf{1 9})$ & 1 & 8 \\
\hline SINUS (3/19) & 2 & 1 \\
\hline \hline & AFS (n= 26) & SINUS (n= 31) \\
\hline P $\max \geq \mathbf{1 2 0}(\mathbf{m s})$ & $12(46)$ & $4(14)$ \\
\hline
\end{tabular}

The values given show numbers of cases with "long P wave" (with percentages in parentheses). $\mathrm{n}=$ number of hearts of particular group with evaluable ECGs. Disregarded were patients with either artificial heart rhythm, or recent myocardial infarction.

key factors capable of modifying myocardial structure and function in AF seem to be atrial tachycardia with a high rate of cell depolarization, and volume/pressure overload of LA leading to increased atrial wall stretch $(3,4)$. These factors are probably responsible for early electrophysiological changes which often precede frank clinical manifestation of AF. In the affected myocardium, the changes are not only electrophysiological but also contractile and particularly structural (1).

These atrial depolarization abnormalities based on atrial myocardial restructuring appear as an arrhythmogenic substrate for development and eventually sustaining of AF. And AF, once established, becomes progressive. It induces further structural changes and worsens the accompanying heart diseases (arterial hypertension, valvular diseases, heart failure etc.), leading to further progression of atrial myocardial injury. This vicious circle is responsible for progression of paroxysmal AF into its more progressive forms $(4,22)$.
The most frequently cited structural change of atrial myocardium is interstitial fibrosis which interferes with atrial conduction $(1,4,21)$. The fibrosis is more or less irreversible, and it seems that the only way how to effectively prevent its formation or progression is antiarrhythmic treatment of AF and treatment of the accompanying heart diseases.

In accordance with findings presented in recent review article by Corradi (4), we showed that patients with AF have significantly more advanced fibrosis in the LA and atrial septum than controls. This, however, did not apply for the RA, where there was no difference in fibrosis between AF and controls. Most severe fibrosis was present in the AFF subgroup, i.e. in patients mostly with a long history of AF, recorded also on the last ECG. This finding is in accordance with results of papers by Kuppahally et al. (13) and by Platonov et al. (20) assessing degree of left atrial myocardial fibrosis in patients with a long history of AF. The former study was based on non-invasive diagnostics by MRI, the latter, like our study, on necropsy histology. Interestingly, Platonov state that in their cohort of patients who died of cardiovascular causes, the extent of fibrosis was not associated with age, but was significantly correlated with AF presence, severity, and duration. They suggest that age-related increase in fibrosis extent does not reach the magnitude of changes observed in AF and age-related changes per se are unlikely to be the sole cause of advanced fibrosis underlying AF.

Another structural change related to development and sustaining of AF is atrial myocardial amyloidosis, in particular the isolated atrial type (IAA). Its precursor - atrial natriuretic peptide (ANP) is normally present in the atrial myocardial interstitium (4).

In our study, we showed that patients with AF, compared to controls, had significantly more severe amyloidosis at all the sites examined. The most severe degree was found in the AFF subgroup. Similar finding was made by Röcken et al., who examined right atrial appendages resected during cardiac surgery (21).

Distribution of amyloid deposits in the atrial walls was irregular, with statistically significant differences. The order of severity was LAA-LAP-LAR. This findings corresponds with that of Šteiner et al. (23), who showed in a study of 100 necropsy patients most severe amyloidosis in the LA anterior wall, particularly in patients with a history of AF. In our study, we also showed different grade of amyloidosis in the atria; the LA was more involved than the RA. This finding corresponds with that of Šteiner et al. (23) and of Leone et al. (14).

Irregular distribution of structural and ultrastructural findings (interstitial fibrosis, distribution of capillaries and cardiomyocyte ultrastuctural changes) in the atrial walls was reported by Corradi et al. $(3,4)$ who showed most advanced findings in the LA posterior wall in comparison with LA appendage.

Other morphological changes followed in our study were heart weight and LA volume. The hearts of patients with AF were significantly heavier (more hypertrophied) than control 
hearts. The heaviest hearts were noted in AFF subgroup. Clinical studies, understandably, evaluate rather LA size and its relation to fixation of $\mathrm{AF}$ and its progression into the more sustained forms. Kerr et al. (12) who studied a group of 757 patients with paroxysmal AF concluded that LA enlargement is an independent risk factor for AF progression into the "chronic form". Similarly Pillarisetti et al. (19) studying a group of 437 patients demonstrated that in patients with valvular disease and enlarged LA paroxysmal AF has a tendency to get fixed. The relation of LA dilatation to AF was documented in a number of clinical studies by means of ultrasound, magnetic resonance or computed tomography. Also large population studies demonstrate that LA size is closely related to AF progression, e.g. the Framingham study, which prospectively followed up adults after routine surveillance M-mode echocardiograms, showed that left atrial size is an independent risk factor for the subsequent development of AF with a hazard ratio of 1.39 for every 5 -mm incremental increase in left atrial size (22). Prospective ultrasound studies showed that AF itself leads to LA dilatation. So, a next vicious circle in etiopathogenesis of $\mathrm{AF}$ is completed (4).

Our findings in necropsy patients correlate with the above cited clinical studies. Patients with AF had significantly larger left atria than controls. The largest atria were observed in patients with AF present on their last ECG record before death (AFF).

To assess a possible relation of structural changes of atrial myocardium to conduction characteristics ( $\mathrm{P}$ wave, QRS complex), we compared the group with most severe amyloidosis (IAA 3) with that with no amyloid detected (IAA 0). There was no difference in either characteristics. This findings is in accordance with that of Ariyarajah et al. (2). On the other hand, Röcken et al. (19), examining RA appendages resected during cardiac surgery in 245 patients, showed that presence of amyloid correlates with $\mathrm{P}$ wave length. He deduced that amyloid deposits interfere with atrial conduction properties.

In our comparison of the IAA 3 vs. IAA 0 we have, however, noticed one interesting finding concerning length of the $\mathrm{P}$ wave. (Normal $\mathrm{P}$ wave length is $110 \mathrm{~ms}$ ). In the IAA 3 group, frequency of the long $P$ wave ( $\geq 120 \mathrm{~ms}$ ) was $35 \%$, while in the IAA 0 it was $25 \%$. Vast majority of these long $\mathrm{P}$ wave cases came from hearts with fibrillation subgroup AFS. When comparing the AFS subgroup (composed primarily of cases with newly diagnosed or paroxysmal AF) and the controls with sinus rhythm, we noticed a marked difference in incidence of long P waves: $46 \%$ in AFS vs. $14 \%$ in controls. Thus, it seems that in patients with AF, who actually present with sinus rhythm, it is more probable that on this ECG there will be an abnormally long P wave than in patients with no history of AF. As we did not find any difference in length of $\mathrm{P}$ wave in patients with severe amyloidosis of the atrial myocardium compared to those with no amyloid, we speculate that elongation of $\mathrm{P}$ wave is due to dilatation of the LA rather than to the structural change.
Again, the question appears, what is the role of IAA in pathogenesis of AF? Seemingly, amyloid is not a strong predictor of $\mathrm{AF}$ and at the same time, AF does not belong to main risk factors for development of amyloid. We must also take into account that IAA is generally much more common than $\mathrm{AF}$ - the prevalence of AF in octogenerians is approximately $10 \%$, while that of IAA at this age is almost $90 \%$. The finding that patients with AF have markedly more severe amyloidosis than control patients with sinus rhythm seems to indicate that the effect of AF on the presence of amyloid has a character of modulation rather than of direct formation. There seems no doubt that IAA is a structural change closely related to AF. Fibrillar forms of ANP induce apoptosis and the amyloid deposits impair contractility and conduction properties of cardiomyocytes. Thus, atrial amyloidosis is yet another of vicious circles accompanying AF. Presence of amyloid predicts AF, and, at the same time, AF increases severity of amyloid deposits (21).

Study limitations. We are aware that particularly the clinical part of our study carries several limitations. Firstly, there is a non-uniformity in classifying the arrhythmia in the clinical documentation. Although in most cases the $\mathrm{AF}$ is clearly specified, there are other with a rather vague statement of "chronic AF", or even no specification of AF at all.

Certain limitation regards also evaluation of the ECG records. We did not take into consideration individual patient's medication with its possible influence on the ECG parameters. And we had to disregard several ECGs because of artifacts, myocardial infarction, or artificial stimulation.

There is also certain limitation regarding the slightly lower average age of control (sinus) patients compared to those of the study (AF) group. However, when comparing certain parameters between study group and more precisely age-matched but in number significantly reduced control group (35 instead 46 subjects), the results were comparable. This fact is consistent with already cited claim of Platonov et al. (20) that atrial myocardial structural abnormalities are related to presence and duration of $\mathrm{AF}$, and not to the patient age. It seems, that lower average age of control patients led also to higher presence of cardiovascular diseases in AF group compared to control group. We are aware of fact, that higher prevalence of systemic hypertension could affect the severity of structural changes of atrial myocardium in AF group. But still, when using for comparison more precisely age-matched reduced control group, the results were comparable. (There were $14(30 \%)$ patients of control group without history of systemic hypertension, when disregarded patient younger than 68 years old than only $3(7 \%)$ patients remained.)

\section{Conclusion}

Our study of necropsy patients showed that patients with a history of atrial fibrillation have, in comparison with those with sinus rhythm, more hypertrophied heart, more volumi- 
nous left atrium, more scarring of left atrial myocardium, and more deposits of isolated atrial amyloid. Distribution of atrial myocardial fibrosis and amyloidosis was irregular; the most affected region was left atrial anterior wall.

Correlation of atrial morphology with ECG records revealed an interesting finding - in patients with more severe atrial amyloidosis and history of atrial fibrillation, with sinus rhythm recorded on their last ECG, there is a tendency for prolongation of the $\mathrm{P}$ wave, when compared with controls. Thus, finding of long $\mathrm{P}$ wave may contribute to diagnosis of a hitherto undisclosed atrial fibrillation.

\section{Acknowledgements}

The authors would like to thank Vignendra Ariyrajah, MD and Jiří Nový, MD for ECG evaluation and RNDr. Eva Čermáková for statistical analysis.

The study was supported by grant BBMRI LM2010004, by Charles University Research Development Schemes (PRVOUK) P37/11 and by Specific Academic Research Projects Competition 266902.

\section{References}

1. Allessie M, Ausma J, Schotten U. Electrical, contractile and structural remodeling during atrial fibrillation. Cardiovasc Res 2002; 54(2): 230-46.

2. Ariyarajah V, Steiner I, Hájková P et al. The association of atrial tachyarrhythmias with isolated atrial amyloid disease: preliminary observations in autopsied heart specimens. Cardiology 2009; 113: 132-137.

3. Casaclang-Verzosa G, Gersh BJ, Tsang TS. Structural and functional remodeling of the left atrium: clinical and therapeutic implications for atrial fibrillation. J Am Coll Cardiol 2008; 51(1): 1-11.

4. Corradi, D. Atrial fibrillation from the pathologist's perspective. Cardiovasc Pathol 2014; 23(2): 71-84.

5. Corradi D, Callegari S, Benussi S et al. Regional left atrial interstitial remodeling in patients with chronic atrial fibrillation undergoing mitral-valve surgery. Virchows Arch 2004; 445(5): 498-505.

6. Corradi D, Callegari S, Benussi S et al. Myocyte changes and their left atrial distribution in patients with chronic atrial fibrillation related to mitral valve disease. Hum Pathol 2005; 36(10): 1080-9.
7. Čihák R, Heinc P, Haman L, Fiala M, Neužil P, Toman O. Fibrilace síní. Cor Vasa 2011; 53(Suppl 1): 27-52

8. De Vos CB, Pisters R, Nieuwlaat R et al. Progression from paroxysmal to persistent atrial fibrillation clinical correlates and prognosis. J Am Coll Cardiol 2010; 55(8): 725-31.

9. Eckstein J, Verheule S, de Groot NM, Allessie M, Schotten U. Mechanisms of perpetuation of atrial fibrillation in chronically dilated atria. Prog Biophys Mol Biol 2008; 97(2-3): 435-51.

10. Haïssaguerre M, Jaïs P, Shah DC et al. Spontaneous initiation of atrial fibrillation by ectopic beats originating in the pulmonary veins. N Engl J Med 1998; 339(10): $659-66$.

11. Kautzner, J et al. Fibrilace síní v běžné praxi. Praha: Maxdorf Jessenius 2012.

12. Kerr CR, Humphries KH, Talajic M et al. Progression to chronic atrial fibrillation after the initial diagnosis of paroxysmal atrial fibrillation: results from the Canadian Registry of Atrial Fibrillation. Am Heart J 2005; 149(3): 489-96.

13. Kuppahally SS, Akoum N, Burgon NS, et al. Left atrial strain and strain rate in patients with paroxysmal and persistent atrial fibrillation: relationship to left atria structural remodelling detected by delayed-enhancement MRI. Circ Cardiovasc Imaging 2010; 3: 231-239.

14. Leone O, Boriani G, Chiappini B et al. Amyloid deposition as a cause of atria remodelling in persistent valvular atrial fibrillation. Eur Heart J 2004; 25(14): $1237-41$.

15. Lloyd-Jones DM, Wang TJ, Leip EP et al. Lifetime risk for development of atrial fibrillation: the Framingham Heart Study. Circulation 2004; 110(9): 1042-6.

16. Luk1, Jan. Fibrilace síní. Praha: Grada Publishing, a.s., 2009.

17. Marchese P, Bursi F, Delle Donne G et al. Indexed left atrial volume predicts the recurrence of non-valvular atrial fibrillation after successful cardioversion. Eur J Echocardiogr 2011; 12(3): 214-21.

18. Matějková A, Šteiner I. Morfologické a elektrofyziologické změny srdečních síní zemřelých s fibrilací síní - pilotní studie. Cesk Patol 2014; 50(4): 150-4.

19. Pillarisetti J, Patel A, Boc K et al. Evolution of paroxysmal atrial fibrillation to persistent or permanent atrial fibrillation: predictors of progression. J Atr Fibrillation 2009; 1(7): 388-394.

20. Platonov PG, Mitrofanov LB, Orshanskaya V, Ho SY. Structural abnormalities in atrial walls are associated with presence and persistency of atrial fibrillation but not with age. J Am Col Cardiol 2011; 58: 2225-32.

21. Röcken C, Peters B, Juenemann G et al. Atrial amyloidosis: an arrhythmogenic substrate for persistent atrial fibrillation. Circulation 2002; 106(16): 2091-7.

22. Saffitz JE, Schuessler RB. Altered atrial structure begets atrial fibrillation, but how? J Cardiovasc Electrophysiol 2004; 15(10): 1175-6.

23. Šteiner I, Hájková P. Patterns of isolated atrial amyloid: A study of 100 hearts on autopsy. Cardiovasc Pathol 2006; 15(5): 287-290.

24. Vaziri SM, Larson MG, Benjamin EJ, Levy D. Echocardiographic predictors of nonrheumatic atrial fibrillation. The Framingham Heart Study. Circulation 1994; 89(2): $724-30$

Received: 18/03/2016 Accepted: 30/05/2016 